\title{
BLOCH FUNCTIONS OF THE THIRD KIND AND THE CONSTANT $\mathfrak{A}$
}

\section{ULUÇAY}

Let $S$ be the class of functions $w=f(z)=z+a_{2} z^{2}+\cdots$ analytic and schlicht for $|z|<1$. For the purpose of obtaining a lower bound on $\mathfrak{A}$ (defined below) Landau [1] considered a subclass $T \subset S$ for which $\left|f^{\prime}(z)\right|\left(1-|z|^{2}\right) \leqq 1$. He showed that $a_{2}=0,\left|a_{3}\right| \leqq 1 / 3$. He also proved the inequality $\mathfrak{A}>.566$. Quite recently E. Reich [2] proved $\mathfrak{A}>.569$. which is very slightly better than Landau's original bound. The purpose of this paper is to give a better bound for $\mathfrak{A}$ and $\left|a_{3}\right|$.

Before proceeding it is convenient to recall the definition of $\mathfrak{A}$ and the definition of a Bloch function of the third kind [3] which belongs to $T$.

Definition. Let $A_{f}$ denote the least upper bound of the radii of all circles contained in the map of $w=f(z)$ which is schlicht for $|z|<1$ and has $\left|f^{\prime}(0)\right|=1$. Then $\mathfrak{A}=\min A_{f}$ is the Landau constant.

DEFinition. If the map of $w=f(z)$ contains no circle of radius greater than $\mathfrak{A}$, then $f$ is called a Bloch function of the third kind.

In the following we shall use the abbreviation B.F. for a Bloch function of the third kind.

THEOREM 1. $\mathfrak{A}>.629$.

Proof. Let $\omega=\zeta+a_{3} \zeta^{3}+\cdots$ be a B.F. then $w=f(z)=z+\left|a_{3}\right| z^{3}$ $+\cdots 1$ is also a B.F. But

$$
|f(z)| \leqq \frac{1}{2} \log \frac{1+|z|}{1-|z|}=|z| M(|z|) .
$$

For arbitrary fixed $s, 0<s<1$, put $f(z, s)=f(s z) / s$. Then

$$
|f(z, s)| \leqq M(s), \quad|z|<1 .
$$

Now write

$$
\bar{f}(z, s, t)=t M(s)\left(\Phi\left((f(z, s) / t M(s))^{3}\right)\right)^{1 / 3}=z+\left|a_{3}\right| s^{2} z^{3}+\cdots,
$$

where $\Phi\left(z^{\prime}\right)=z^{\prime} /\left(1 \pm t z^{\prime}\right)^{2}, t>0,\left|z^{\prime}\right|<1 / t$. If $f(z)$ omits $c$, then $\tilde{f}(z, s, t)$ omits

$$
\gamma(s)=(c / s)\left(1 \pm t^{-2} c^{3} / s^{3} M^{3}(s)\right)^{-2 / 3} .
$$

Received by the editors December 7, 1956 and, in revised form, December 27, 1956.

1 Obtained by rotating the $\omega$ and $\zeta$-plane through an angle equal to $-2^{-1}$ arg $a_{3}$. 
If $-\pi / 2 \leqq \arg c^{3} \leqq \pi / 2$, we take the sign + . For a suitable choice of $t$, $\gamma(s)$ is real, $>0$ and $s \gamma(s) \leqq|c|$. In the alternative case, we take the sign - and replace $c^{3}$ by $-c^{3}$. Again for a suitable choice of $t, \gamma(s)$ is real and $(<0)$ and $s|\gamma(s)| \leqq|c|$. Thus in all cases, for a proper choice of $t$,

$$
F(z, s)=\frac{\gamma(s) \tilde{f}(z, s, t)}{\gamma(s)-\tilde{f}(z, s, t)}=z+b_{2} z^{2}+b_{3} z^{3}+\cdots,
$$

where $b_{2}=1 / \gamma(s), b_{3}=1 / \gamma^{2}(s)+\left|a_{3}\right| s^{2}$, is schlicht for $|z|<1$ and omits $\gamma(s)$ real ( $\$ 0$ according to the cases).

Now, since $F(z, s)$ is analytic and schlicht for $|z|<1$, then $[4 ; 5]$

I.e.,

$$
\left|b_{3}-\alpha b_{2}^{2}\right| \leqq 2 e^{-2 \alpha /(1-\alpha)}+1, \text { for all } 0<\alpha<1 \text {. }
$$

$$
\left|a_{3}\right| s^{2}+(1-\alpha) / \gamma^{2}(s) \leqq 2 e^{-2 \alpha /(1-\alpha)}+1 \text {. }
$$

Hence

$$
(1-\alpha) / \gamma^{2}(s) \leqq 2 e^{-2 \alpha /(1-\alpha)}+1, \quad \text { for all } 0<\alpha<1 .
$$

In particular for $\alpha=.45$, a numerical computation yields

$$
|\gamma(s)|>\text {.629. }
$$

If $c$ is nearest to the origin then $\mathfrak{A} \geqq|c|$. Hence $.629 s<s|\gamma(s)| \leqq|c|$ $\leqq \mathfrak{A}$, i.e., $.629 s<21$. Making $s \rightarrow 1$ we have the result.

Theorem 2. If $f$ is a B.F. and belongs to $T$, then $\left|a_{3}\right|<1 / 9$.

Proof. (1) and $s|\gamma(s)| \leqq \mathfrak{A}$ imply

$$
s^{2}\left(\left|a_{3}\right|+(1-\alpha) \mathfrak{H}^{-2}\right) \leqq 2 e^{-2 \alpha /(1-\alpha)}+1,
$$

and in particular for $s \rightarrow 1$

$$
\left|a_{3}\right|+(1-\alpha) \mathfrak{A}^{-2} \leqq 2 e^{-2 \alpha /(1-\alpha)}+1 .
$$

In particular for $\alpha=1 / 2, \mathfrak{A}<.6565$ [6], and a numerical computation yields the result stated in the theorem.

It is very likely that $a_{3}=0$ and that the map of the corresponding B.F. is three-fold symmetric.

\section{REFERENCES}

1. E. Landau, Über die Blochsche Konstante . . MZ. vol. 30 (1929).

2. E. Reich, On a Bloch-Landau constant, Proc. Amer. Math. Soc. vol. 7 no. 1 (1956).

3. R. M. Robinson, Bloch functions, Duke Math. J. vol. 2 (1936).

4. A. C. Schaeffer and D. C. Spencer, Coefficient regions for schlicht functions, Amer. Math. Soc. Colloquium Publications, vol. 35, 1950. 
5. G. M. Goluzin, Some questions of the theory of univalent functions, Trudy Mat. Ins. Steklov, vol. 27 (1949).

6. Ruth E. Goodman, On the Bloch-Landau constant for schlicht functions, Bull. Amer. Math. Soc. vol. 51 (1945) pp. 234-239.

ANKaRA UNIVERSITY

\section{A T-SYSTEM WHICH IS NOT A BERNSTEIN SYSTEM ${ }^{1}$}

\section{RICHARD G. LONG}

(I) Let $E$ be a separable real Banach space, let $\left\{u_{i}\right\}_{1}^{\gamma}$ be a fundamental ${ }^{2}$ linearly independent sequence (finite or infinite) of points of $E$, and for each $0 \leqq n<\gamma+1$, let $L_{n}$ be the linear extension of $\left\{u_{i}\right\}_{1}^{n}$, with $L_{0}=\{\phi\}$. Then, $\left\{u_{i}\right\}_{1}^{\gamma}$ is called a $T$-system for $E$ if for each $x$ in $E$ and for each $0 \leqq n<\gamma+1$ there is a unique $P_{n}(x)=\sum_{i=1}^{n} x_{i, n} u_{i}$ in $L_{n}$ such that $\left\|x-P_{n}(x)\right\|=\inf _{Q \in L_{n}}\|x-Q\|$. The deviation sequence, $\left\{\delta_{i}(x)\right\}_{0}^{\gamma}$, of $x$ relative to $\left\{u_{i}\right\}_{1}^{\gamma}$ is defined by the conditions: (1) $\left|\delta_{i}(x)\right|=\left\|x-P_{i}(x)\right\|$ for all $0 \leqq i<\gamma+1$ and (2) for $0 \leqq i<\gamma+1$, if $\delta_{i}(x) \neq 0$, then $\operatorname{sgn} \delta_{i}(x)=\operatorname{sgn} x_{i^{\prime}, i^{\prime}}$, where $i^{\prime}$ is the least integer greater than $i$ for which $x_{i^{\prime}, i^{\prime}} \neq 0$. If $\left\{\delta_{i}(x)\right\}_{0}^{\gamma} \equiv\left\{\delta_{i}(y)\right\}_{0}^{\gamma}$ implies that $x=y$, then the $T$-system $\left\{u_{i}\right\}_{1}^{\gamma}$ is called a Bernstein system for $E$.

The definitions of $T$-systems and Bernstein systems were essentially introduced by Kadeč in [4], in generalizing some results of Bernstein [2]. The proofs in [2] and [4] show or can be used to show that any two separable Banach spaces are homeomorphic if they possess Bernstein systems of the same cardinality and that for finite dimensional spaces every $T$-system is a Bernstein system. Now, given a separable Banach space we can always find a fundamental linearly independent sequence of points in it and by strictly convexifying the space [3] such a sequence becomes a $T$-system. In [4], Kadeč states as an open question: Is every $T$-system a Bernstein system? Since every separable infinite dimensional Banach space has a $T$-system under some equivalent norm, an affirmative answer to this would have proved that all such spaces are homeomorphic.

It is the purpose of this paper to exhibit a $T$-system which is not a Bernstein system. In (II), we describe such a system for the space $c_{0}$ under a norm equivalent to the usual sup norm.

We note that Kadeč [4] showed that $c_{0}$ does possess a Bernstein

Presented to the Society December 29, 1956; received by the editors December 19, 1956.

1 This research was supported in part by the National Science Foundation.

2 Most terms not defined in the text may be found in Banach [1]. 